\title{
Supermassive Black Holes Then and Now
}

\author{
D. Richstone \\ Institute of Advanced Study, Princeton, \\ and Dept. of Astronomy, University of Michigan
}

\begin{abstract}
Recent surveys suggest that most or all normal galaxies host a massive black hole with $1 / 100$ to $1 / 1000$ of the visible mass of the spheroid of the galaxy. Various lines of argument suggest that these galaxies have merged at least once in our past lightcone, and that the black holes have also merged. This leads to a merger rate of massive black holes of about $1 / \mathrm{yrs}$.
\end{abstract}

\section{INTRODUCTION}

Supermassive black holes have been a prime candidate for the probable energy sources of quasars, the most energetic objects in the universe, since the discovery of quasars. Over the last decade local surveys have suggested that quasars are present in most galaxies in the present universe $[1,2]$. The demographics of these objects are so fundamental to an estimate of their merger rate that we repeat the key points below.

Except where noted, All quantities in this paper are computed for a Friedman-Robertson-Walker Universe with $\Omega=1$ and $H_{0}=80 \mathrm{~km} \mathrm{~s}^{-1} \mathrm{Mpc}^{-1}$. Distances to nearby MBHs come from many sources, but are always rescaled to this Hubble constant.

\section{STATISTICS OF MASSIVE BLACK HOLES IN GALAXY CENTERS}

In about 15 cases, high resolution spectroscopy and imaging, coupled with detailed modelling has led to clear evidence for the presence of a massive dark object (MDO) in the centers of nearby galaxies (including our own). The common denominator in all these studies is the identification of test particles (stars or gas clouds), which orbit the object of mass $(M)$ at a distance $r$ at a speed $v$ given by $v^{2}=\alpha G M / r$. The estimate of $\alpha$ requires a detailed model, but often $\alpha \sim 1$. In the fortunate cases of a disk of stars or gas, the analysis is straightforward and fairly unambiguous. In the more complicated case of an anisotropic distribution of stellar orbits it is necessary to construct a detailed model. The favored technique is based on orbit superposition and is summarized in a number of articles $\{3,4]$. These well-defined cases are listed in $[1]$ and labelled in Figure 1.

In three cases (NGC 4258, the Galaxy and M32), it is possible to reject many alternatives to a black hole (such as clusters of neutron stars or black holes) for the observed MDO. The basic argument $[5,6]$ against aggregate models is that the requirement that the evaporation time be less than the probable system age (a Hubble time) sets an upper limit on the mass of the constituent objects that in these cases is near $0.1 M_{\odot}$. Brown dwarfs or planets (or white dwarfs) of this mass or less would rapidly merge. There are no known stellar remnants of any sort of this mass. The MDO's might be clusters of low mass black holes or uninteracting elementary particles (of an unknown variety), but the formation of the former and the collapse of clusters of either to a dense state would both require major new theories. Based on these three objects, for the rest of this paper we assume that all MDO's are massive black holes (bh).

In addition to these very carefully studied cases, we [7] have combined HST images with ground-based spectra to analyze another 20 objects using two-integral distribution function based methods (this is inherently riskier than the orbit superposition methods used for the better data). Combining these analyses suggest that every normal galaxy has a massive black dark object at the present epoch, and that the black hole mass is proportional to the bulge mass of the galaxy (the visible mass of the entire galaxy if the galaxy is an elliptical). The relation between bh mass and the bulge luminosity is $M_{\bullet}=2 \times 10^{7}\left(L_{\text {bulge }} / 5 \times 10^{9} L_{\odot}\right)^{1.2}$. 


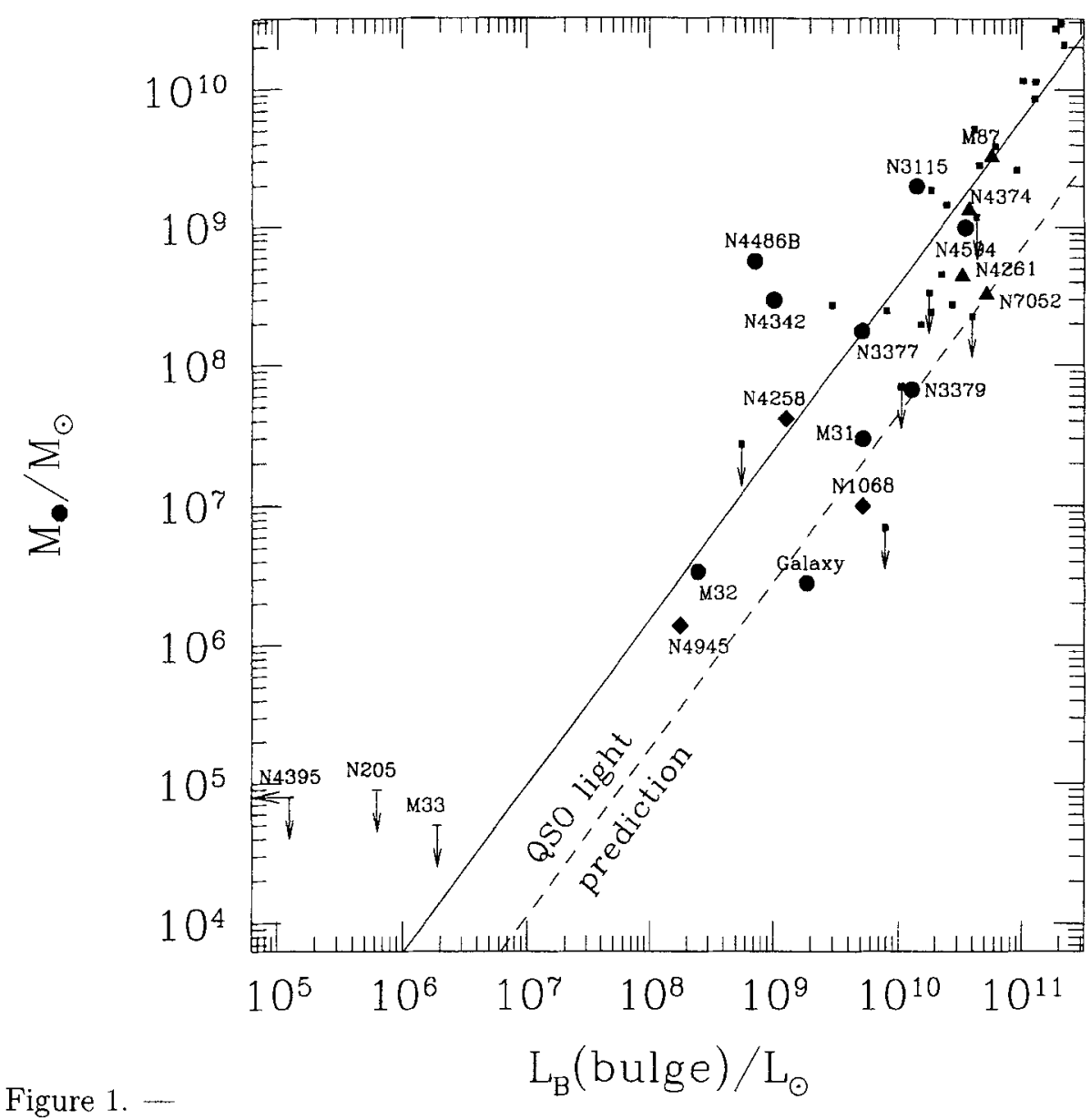

Figure 1.

Mass estimates of the candidate MBHs in galaxies with dynamical information plotted against the bulge luminosity of their host galaxy. The labeled points are the results of painstaking observation and detailed modelling. The symbols indicate the how $M_{\text {. }}$ was derived: kinematics of gas - triangles; dynamics of stars - filled circles; masers - diamonds; or two-integral modelling using ground-based stellar kinematics -- small squares. Arrows indicate upper limits on $M_{\bullet}$. The solid line is a model with $M_{\bullet}=0.005 M_{\text {bulge }}$ and $M_{\text {bulge }}=$ $5 \times 10^{9} M_{\odot}\left(L_{\text {bulge }} / 10^{9} L_{\odot}\right)^{1.2}$. The distribution of $M_{\bullet}$ is roughly Gaussian in $\log \left(M_{\bullet} / M_{\text {bulge }}\right)$ with mean -2.27 $\left(M_{\bullet} / M_{\text {bulge }}=0.005\right)$ and standard deviation 0.5 . The dashed line is the quasar light prediction of eqn 3 apportioned according to the bulge mass: $M_{\bullet}=2 \times 10^{7}\left(L_{\text {bulge }} / 5 \times 10^{9} L_{\odot}\right)^{1.2}$.

The small offset from the observed black-hole/bulge-mass relation indicates that the present integrated density in MBHs is broadly consistent with the integrated luminosity produced by AGNs over the life of the Universe. This offset may reflect a radiative efficiency of average quasar accretion less than 0.10 . This figure is reproduced from reference 1. 
Because quasars were populous in the youthful Universe, but have mostly died out, the Universe should be populated with relic black holes whose average mass density $\rho_{u}$ matches or exceeds the mass-equivalent of the energy density $u$ emitted by them [8]. The integrated comoving mass-equivalent density in quasar light (as emitted) is

$$
\rho_{u}=1 /\left(\epsilon c^{2}\right) \int_{0}^{\infty} \int_{0}^{\infty} L \Phi_{Q}(L \mid z) d L \frac{d t}{d z} d z=2 \times 10^{5}\left(\frac{0.1}{\epsilon}\right) M_{\odot} \mathrm{Mpc}^{-3} .
$$

where $\Phi_{Q}$ is the comoving density of quasars of luminosity $L$, and $t$ is cosmic time and $\epsilon$ is the radiative efficiency. This density can be compared to the luminous density in galaxies. Using Loveday's estimates of the parameters of a Schechter luminosity function

$$
\Phi_{G}(L) d L=\phi^{*}\left(\frac{L}{L^{*}}\right)^{-1} e^{-L / L^{*}} d\left(\frac{L}{L^{*}}\right)
$$

with $\phi^{*}=1.4 \times 10^{-2} h^{3} \mathrm{Mpc}^{-3}$ gives a luminous density of $j=1.1 \times 10^{8} L_{\odot} \mathrm{Mpc}^{-3}$ [9], we obtain the ratio of the mass in relic MBHs to the light of galaxies $(h=0.8)$ :

$$
\Upsilon=\frac{\rho_{u}}{j}=1.8 \times 10^{-3}\left(\frac{0.1}{\epsilon}\right)\left(\frac{M_{\odot}}{L_{\odot}}\right) .
$$

We can compare the estimate of [7] to the prediction of the total luminosity in quasars (above) by apportioning the quasar-predicted mass according to the mass of each galaxy. The quasar light underpredicts the observed black hole masses by about a factor of 5 , suggesting that a large fraction of black hole growth may occur at radiative efficiencies significantly less than 0.1 .

\section{AN ATTEMPT TO QUANTIFY THE MERGER RATE}

The previous section suggests that every galaxy hosts a massive black hole. In the hierarchical model of galaxy formation elliptical galaxies form and grow as a result of generations of mergers of comparably massive progenitors. The exact nature of the progenitors and the epoch of the mergers are both uncertain, but several lines of argument suggest that there is a high merger rate of galaxies containing bh's in our past light cone.

The number density of galaxies above a luminosity of $0.01 L^{*}$ is (from eqn 2)

$$
n_{0.01}=\phi^{*} \int_{0.01}^{\infty} x e^{-x} d x=5.6 \times 10^{-2} h^{3} \mathrm{Mpc}^{-3}
$$

Multiplying this by the Hubble volume $4 \pi c^{3} /\left(3 H_{0}^{3}\right)$ gives an estimate of $6 \times 10^{9}$ galaxies in our past lightcone. Dividing by $t_{0}=8 \times 10^{9} \mathrm{yrs}$ gives a merger rate since redshift $z=1$ of $0.7 \mathrm{hyrs}^{-1}$, if each galaxy undergoes one merger in that time. One might expect a comparable contribution to the merger rate from higher redshifts, at least up to $z \sim 3$ where the quasars are most numerous, suggesting that the massive bh population formed then or earlier [1]. There are several lines of argument that this merger rate is reasonable. The simplest approach to a galaxy merger rate is to use the Press-Schechter [10] formalism to estimate the change in the number of objects at a mass of about $10^{12} M_{\odot}$ since $z=3$. For a fairly standard normalization of $\sigma_{8}=1$ at present and a bias near $1[11]$, the number of collapsed objects has increased by about a factor of order unity in an $\Omega_{0}=1$ cosmology, and about $1 / 3$ in a $\Omega_{0}=.2$ Universe. The merger rate at higher redshifts is higher. A better calculation based on semi-analytic galaxy formation models and "conditional" Press-Schechter formalism suggests a growth factor of $\sim 10[12]$ since $z=3$.

A second argument can be made from the observations of the "Lyman break objects", which suggests that the brightest objects seen at $z \sim 3$ are a factor of 10 less massive than bright objects today and considerably more numerous [13-15].

If the galaxies merge, do the massive black holes contained in them merge as well? Many of the calculations needed to answer this question have been carried out in a somewhat different context [16]. Dynamical friction will carry a massive black hole of $10^{7} M_{\odot}$ or more into the center of a host galaxy in less than a Hubble time from far out in the galaxy. Smaller black holes, cloaked in sufficient numbers of bound stars from their parent pre-merger galaxy, will similarly be carried to the center. Two massive black holes in the center of such a galaxy will form a hard binary which decays increasingly slowly due to stellar scattering, until gravitational 
radiation becomes important. For galaxies with central densities like the Milky Way, black holes more massive, than $10^{6} M_{\odot}$ will reach a high enough binding energy to decay by gravitational radiation in less than a Hubble time. A similar look at this problem in a variety of galaxy types would be valuable.

Finally, there is some observational information that can be brought to bear on the question of mergers of black holes in our past light cone. Although the observed mass density of supermassive black holes is only 5 times greater than that predicted from the integral of the quasar light, the number of black holes of $10^{8} M_{\odot}$, corresponding to Eddington luminosities of $\sim 10^{46} \mathrm{ergs} / \mathrm{sec}$, is about $10^{-3} \mathrm{Mpc}^{-3}$ [1], while the number of quasars with luminosities of $10^{46} \mathrm{ergs} / \mathrm{sec}$, at the peak of quasar numbers at $z \sim 3$ is only about $10^{-6} \mathrm{Mpc}^{-3}$ [17]. This discrepancy can be resolved in one of two ways. The obvious one is that quasars shine only for about $10^{6} \mathrm{yrs}$. This seems implausible as they could then only accrete (even at super Eddington rates) a few percent of their mass in this time, and must gain the rest in a manner invisible to us. Alternatively, they may have merged a few times since the quasar era. Even two generations of merging (producing a factor of 4 change in mass of a typical black hole since the quasar epoch) goes a long way to resolving the "numbers crisis" because we must then identify the quasars that powered the bright quasars at early epochs with much more massive black holes today. Since the galaxy luminosity function (and by hypothesis, the bh mass function) falls exponentially at high mass this modest growth factor serves to bring the numbers at high and low redshift into line (see [1]).

Thus it seems likely on several grounds that the supermassive black hole population has undergone a few mergers since the quasar epoch. If this is so, the bh merger rate in our lightcone could easily exceed $1 / \mathrm{yrs}$. As noted in other talks at this meeting, these mergers should be observable for masses of at least $10^{6} M_{\odot}$. Since our best current understanding is of yet higher mass black holes, it would be desirable to maintain or improve LISA's performance at the lowest frequency, where the heaviest objects will radiate. On the other hand, LISA may give us the best handle on the mergers of the low mass objects, and may provide the only information we will get on the mergers of protogalaxies before $z \sim 3$.

I'm grateful to the "Nukers" (E. A. Ajhar, R. Bender, G. Bower, A. Dressler, S. M. Faber, A. V. Filippenko, K. Gebhardt, R. Green, L. C. Ho, J. Kormendy, T. Lauer, J. Magorrian \& S. Tremaine) and aiso to John Bahcall, Pawan Kumar, J. P., Ostriker, Martin Rees and David Spergel, for discussions of these topics. I thank the Ambrose Monell Foundation, the Guggenheim Foundation and NASA for financial support.

\section{REFERENCES}

1. Richstone, D., et al. Nature, in press, Oct 1998.

2. Kormendy, J. \& Richstone, D. Inward bound - the search for massive black holes in galactic nuclei. Ann Rev Astron and Astroph 33, 581 - 624 (1995).

3. Richstone, D. O. \& Tremaine, S. ApJ 327, $82--88$ (1988).

4. van der Marel, R. P., Cretton, N., de Zeeuw, P. T. \& Rix, H.-W. ApJ 493, 613-631 (1998).

5. Goodman, J. \& Lee, H.-M. ApJ 337, $84-90$ (1989).

6. Maoz, E. ApJ Letters 447, L91 - L94 (1995). Updated in Maoz, E. ApJ Letters 491, L181 - 184 (1998).

7. Magorrian J. et al. AJ 115, in press (1998).

8. Chokshi A. and Turner, E. L. MNRAS 259, $421-424$ (1992).

9. Loveday, J., Peterson, B. A., Efstathiou, G. and Maddox, S. J. ApJ 390, 338 - 344 (1992).

10. Press, W. H. \& Schechter, P. L. ApJ 187, 425 (1974).

11. Strauss, M. A., Willick, J. A. Phys Reports, 261, $271-431$ (1995).

12. Somerville, R., Primack, J. \& Faber, S.M. ApJ in press (1998).

13. Steidel, C. C., Giavalisco, M., Pettini, M., Dickinson, M. \& Adelberger, K. L. ApJ Letters 462, L17 - L21 (1996).

14. Lowenthal, J.D. et al. ApJ 481, 673-688 (1997).

15. Trager, S.C., Faber, S.M., Dressler, A. \& Oemler, A. ApJ 485, 92 - 99 (1997).

16. Xu, G. \&Ostriker, J. P. ApJ 437, 184 (1994).

17. Schmidt, M., Schneider, D. P. \& Gunn, J. E. AJ 110, $68-77$ (1995). 